\title{
AIDS and behavioural risk factors in women in inner city Baltimore: a comparison of telephone and face to face surveys
}

\author{
Manel Nebot, David D Celentano, LaWanda Burwell, Aria Davis, Mary Davis, \\ Michele Polacsek, John Santelli
}

\begin{abstract}
Study objective - The study aimed to investigate the influence the mode of administration of a questionnaire (telephone or face to face) on reports of sexual behaviour and attitudes of HIV risk among woman of reproductive age.

Design - Two cross sectional surveys one, a modified random digit dialing telephone survey, the second, a face to face street sample - were carried out by the same interviewers using similar questionnaires in the same neighbourhoods.
\end{abstract}

Setting and participants - Two socially deprived, inner city neighbourhoods of Baltimore City were assessed in early 1990 before a community health intervention was carried out in one of them. Women between 17 and 35 years were surveyed.

Main results - Altogether 775 and 416 women in the target age group were interviewed by telephone and face to face methods: the response rates were $66.4 \%$ and $77 \%$ respectively. Telephone respondents tended to be older, had more education, were more often married, were less likely to live in subsidised housing, and were more likely to report HIV testing. The proportions of respondents who reported a previous abortion and had had a surgical sterilisation were higher among the telephone respondents (34.7\% $v 24 \cdot 1 \%$ and $26 \cdot 4 \%$ v $20 \cdot 6 \%$, respectively). With regard to sexual risk behaviour, the only statistically significant differences were found in the proportion who reported having used drugs $(10.6 \%$ of the face to face $v \mathbf{2 . 4 \%}$ of the telephone sample) or alcohol (30.5\% v 16.3\%) at last sexual intercourse. The observed method effect on these variables remained unchanged after adjusting for age, education, employment, and marital status. This effect was even stronger for a subgroup of face to face respondents who reported not having a telephone at home. The adjusted odds ratios for reporting alcohol consumption and use of drugs at the last sexual encounter in this group compared with the telephone respondents were $3 \cdot 7(2 \cdot 1,6 \cdot 6)$ and $14 \cdot 1(5 \cdot 7,34 \cdot 5)$ respectively.

Conclusions - Despite the socioeconomic bias associated with the mode of data collection, there are only a few differences between the telephone and personal survey methods in reports of sexual behaviour. These differences are mostly concentrated in young women (under 20 years), and in a particulary socioeconomically deprived subgroup identified through telephone ownership.

\section{( E Epidemiol Community Health 1994;48:412-418)}

Telephone surveys have been widely used for more than three decades in health survey research. By 1960 , approximately $80 \%$ of all United States households had a telephone, a proportion that had increased to $90 \%$ by $1970 .^{1}$ There is evidence that telephone surveys, despite their tendency to reach a younger, better educated sample, yield basically the same information as personal interviews, ${ }^{2-4}$ at considerably less expense. Yet, there are some differences in health, outcomes obtained from telephone and personal interviews that are attributable to differences in the survey method. These differences are complex and not limited solely to the interviewing process. Firstly, telephone surveys have biases of both coverage and response, leading to oversampling the more affluent, more educated population. ${ }^{35}$ Telephone respondents have also been reported to be impatient to complete interviews and to avoid answers that may lengthen the interview. ${ }^{4}$ In addition, telephone surveys tend to have more missing data, ${ }^{3}$ which can in turn attenuate some of the expected relationships between the independent and dependent variables. ${ }^{6}$

With regard to the assessment of sensitive topics, such as the use of drugs or sexual behaviour, there is little evidence to date to support any clear advantage of either personal or telephone interviews for most health survey research. Coombs ${ }^{7}$ reported that sensitive data on family planning and pregancy could be reliably obtained using telephone interviews on a sample who had previously completed a face to face interview. However, Czaja ${ }^{8}$ found that telephone respondents reported less frequently socially disapproved sexual behaviours, such as having a large number of sexual partners. These findings were not replicated by McQueen, ${ }^{9}$ who observed that more men reported high risk sexual behaviours, such as having a greater number of partners or having had sex with other men, in telephone 
interviews. This suggests that the greater anonymity associated with telephone interviews compared with personal contact, could yield more frequent reports of risky, socially disapproved behaviours.

The data on the differential reporting of sensitive behaviour obtained during telephone and face to face interviews do not therefore seem to be conclusive at this point. As a growing number of HIV/AIDS prevention programmes base their needs assessment and evaluations on empirical data, it is important to be aware of methodological biases that might exist in data collection. ${ }^{10}$ To address this issue, we present here details of self reported sexual behviour and attitudes related to the risk of HIV infection among woman of reproductive age in two inner city areas of Baltimore City, assessed both through a telephone and a face to face interview. These surveys were designed as a baseline assessment for a communty based education project, which focused on reducing the risk of HIV infection through sexual relations, basically by encouraging the use of condoms among the target populations described above.

\section{Methods}

We developed a pre-intervention assessment for a project that aimed to reduce the risk of perinatal transmission of $\mathrm{HIV}$ infection among women of reproductive age in two, socially disadvantaged, inner city neighbourhoods of Baltimore, Maryland. A telephone and a personal interview were carried out in intervention and comparison neighbourhoods. Both surveys used the same questionnaire and undertaken sequentially between December 1989 and May 1990. The primary target population was defined as women between 17 and 35 years old. Women aged less than 17 years were not interviewed because of the difficulties in obtaining parental permission, and those aged 35 years and more were not approached because this is the approximate ending point of parity used in other similar populations. ${ }^{11}$

The questionaire was developed from previous instruments used in AIDS research, and was piloted before the field study to select and tailor the items to the target population. ${ }^{12}$ Measures of internal consistency were used to assess reliability in the questionnaire, although no "gold standard" measure exists for ascertaining validity. Knowledge of HIV transmission and perceived community norms were measured using five point Likert scales. ${ }^{13}$ In addition, demographic variables, pregnancy antecedents, and contraception practices, as well as HIV related risk behaviours, including drug use and sexual behaviour were ascertained.

A Waksberg ${ }^{14}$ method of random digit dialling was undertaken in the telephone survey, which utilises screened telephone numbers (household telephone numbers in service) to form the initial primary sampling unit. Once a residential telephone has been identified in the target community, two new numbers are created replacing the final two digits of the screened telephone number. To minimise non-response, all initial refusals were telephoned again two weeks after the first call, and a minimum of 10 calls was made before closing a number as a "no answer". Seven trained female interviewers made the telephone calls from a centralised facility between 4 and $9 \mathrm{pm}$, Monday to Thursday, between December 1989 and April 1990. Calls were also made on Saturday morning to those numbers for which no response had been achieved during the weekday calls. In a random subsample of the telephone questionnaires, other domains such as the relationship between attitudes and social norms were explored, ${ }^{12}$ and some of the core questions were therefore omitted in this version of the questionnaire. This includes the self reported antecedents of abortion, use of drugs, and use of alcohol at last sexual intercourse that were omitted for one third of the telephone questionnaires. On the other hand, some questions, such as living in publicly subsidised housing, were present in the in depth version only, and so were addressed to only about one third of telephone respondents.

A street outreach, targeted sampling method was chosen for the face to face survey. This is a convenient method when random sampling is neither possible nor desirable. ${ }^{15}$ The interview began immediately after the telephone survey finished (May 1990), and was completed in about two months. Using this method the communities were divided into small sections of a few blocks each to create geographical zones of comparable population density. Special attention was given to maintaining consistency within each geographical zone in the selection of participants so that samples were similar in terms of age and gender. A total of seven African-American women were trained to sample potential respondents and to administer the interview in the street after subjects had been screened for eligibility (age between 17 and 35, living in the neighbourhood, and having not completed a previous survey from our team), and had given voluntary informed consent. Each day three pairs of interviewers were assigned to three different sectors, to cover the entire geographical area in each community, and approached all those women who were not obviously out of the desired age range. In addition to the conventional $\chi^{2}$ test for homogeneity, ${ }^{16}$ unconditional logistic regression ${ }^{17}$ was used to obtain odds ratios adjusted for demographic variables when comparing reported risk behaviours in the samples.

\section{Results}

A total of 1837 households in the target and control communities was reached between December 1989 and April 1990 by the telephone interviewers. Out of these, there were 445 refusals before elegibility could be ascertained, and 173 refusals in eligible people. There were, therefore, 1219 valid responses (response rate $66 \cdot 4 \%)$. Of these, $775(63.4 \%)$ were women. In the following three months, 416 interviews were completed by eligible 
Table 1 Selected demographic and HIV related risk factors among the survey's female respondents in relation to interview mode and telephone ownership

\begin{tabular}{|c|c|c|c|c|c|c|}
\hline & $\begin{array}{l}\text { Telephone } \\
\text { interview }\end{array}$ & & $\begin{array}{l}\text { Face to } \\
\text { intervie }\end{array}$ & total) & $\begin{array}{l}\text { Face to } \\
\text { telepho }\end{array}$ & $\begin{array}{l}e \text { (no } \\
\text { wners) }\end{array}$ \\
\hline & & $\left(N_{0}\right)^{*}$ & & $(\mathrm{No}) \dagger$ & & $(\mathrm{No})$ \\
\hline $\begin{array}{l}\text { Age }(y): \\
<20 \\
20-24 \\
25-29 \\
30-35\end{array}$ & $\begin{array}{l}14 \cdot 8 \\
22 \cdot 0 \\
25 \cdot 5 \\
37 \cdot 7\end{array}$ & $\begin{array}{l}(114) \\
(170) \\
(197) \\
(290)\end{array}$ & $\begin{array}{l}21 \cdot 4 \\
36 \cdot 7 \\
24 \cdot 8 \\
17 \cdot 1\end{array}$ & $\begin{array}{l}(89) \\
(152) \\
(103) \\
(71)^{* *}\end{array}$ & $\begin{array}{l}11 \cdot 4 \\
38 \cdot 0 \\
36 \cdot 7 \\
13 \cdot 9\end{array}$ & $\begin{array}{l}(9) \\
(30) \\
(29) \\
(11) \dagger \dagger\end{array}$ \\
\hline $\begin{array}{l}\text { Highest school grade: } \\
\text { None } \\
\text { Elementary } \\
\text { High school } \\
\text { College/graduate }\end{array}$ & $\begin{array}{r}0 \cdot 5 \\
19 \cdot 6 \\
72 \cdot 6 \\
7 \cdot 2\end{array}$ & $\begin{array}{r}(4) \\
(152) \\
(563) \\
(56)\end{array}$ & $\begin{array}{r}1 \cdot 2 \\
35 \cdot 9 \\
61 \cdot 2 \\
1 \cdot 7\end{array}$ & $\begin{array}{l}(5) \\
(147) \\
(250)^{* *} \\
(7)\end{array}$ & $\begin{array}{r}3 \cdot 8 \\
48 \cdot 1 \\
45 \cdot 6 \\
2 \cdot 5\end{array}$ & $\begin{array}{l}(3) \\
(38) \\
(36)^{* *} \\
(2)\end{array}$ \\
\hline $\begin{array}{l}\text { Ethnicity: } \\
\text { African American } \\
\text { Other }\end{array}$ & $\begin{array}{r}90 \cdot 4 \\
9 \cdot 6\end{array}$ & $\begin{array}{r}(697) \\
(74)\end{array}$ & $\begin{array}{r}92 \cdot 7 \\
7 \cdot 3\end{array}$ & $\begin{array}{r}(382) \\
(30)\end{array}$ & $\begin{array}{l}88 \cdot 3 \\
11 \cdot 7\end{array}$ & $\begin{array}{r}(68) \\
(9)\end{array}$ \\
\hline $\begin{array}{l}\text { Martial status: } \\
\text { Never married } \\
\text { Married } \\
\text { Other }\end{array}$ & $\begin{array}{l}63 \cdot 5 \\
21 \cdot 1 \\
15 \cdot 3\end{array}$ & $\begin{array}{l}(492) \\
(164) \\
(119)\end{array}$ & $\begin{array}{r}68 \cdot 0 \\
9 \cdot 2 \\
22 \cdot 8\end{array}$ & $\begin{array}{l}(281) \\
(38) \\
(94)^{* *}\end{array}$ & $\begin{array}{r}72 \cdot 2 \\
5 \cdot 1 \\
22 \cdot 7\end{array}$ & $\begin{array}{r}(57) \\
(4) \\
(18)\end{array}$ \\
\hline $\begin{array}{l}\text { Work outside home: } \\
\text { No } \\
\text { Yes }\end{array}$ & $\begin{array}{l}35 \cdot 2 \\
64 \cdot 8\end{array}$ & $\begin{array}{l}(271) \\
(500)\end{array}$ & $\begin{array}{l}54 \cdot 1 \\
45 \cdot 9\end{array}$ & $\begin{array}{l}(214) \\
(182)^{* *}\end{array}$ & $\begin{array}{l}74 \cdot 0 \\
26 \cdot 0\end{array}$ & $\begin{array}{l}(57) \\
(20)^{* *}\end{array}$ \\
\hline $\begin{array}{l}\text { Public housing §: } \\
\text { No } \\
\text { Yes }\end{array}$ & $\begin{array}{l}62 \cdot 3 \\
37 \cdot 7\end{array}$ & $\begin{array}{r}(160) \\
(97)\end{array}$ & $\begin{array}{l}43 \cdot 2 \\
56 \cdot 8\end{array}$ & $\begin{array}{l}(198) \\
(218)^{* *}\end{array}$ & $\begin{array}{l}23 \cdot 1 \\
76 \cdot 9\end{array}$ & $\begin{array}{l}(18) \\
(60)^{* *}\end{array}$ \\
\hline $\begin{array}{l}\text { Ever had sex: } \\
\text { No } \\
\text { Yes }\end{array}$ & $\begin{array}{r}4 \cdot 7 \\
95 \cdot 3\end{array}$ & $\begin{array}{r}(35) \\
(716)\end{array}$ & $\begin{array}{r}8 \cdot 3 \\
91 \cdot 7\end{array}$ & $\begin{array}{c}(34) \\
(375)+\dagger\end{array}$ & $\begin{array}{r}7.7 \\
92 \cdot 3\end{array}$ & $\begin{array}{l}(6) \\
(72)\end{array}$ \\
\hline $\begin{array}{l}\text { IVDU last month: } \\
\text { No } \\
\text { Yes }\end{array}$ & $\begin{array}{r}98 \cdot 7 \\
1.3\end{array}$ & $\begin{array}{r}(765) \\
(10)\end{array}$ & $\begin{array}{r}98.5 \\
1.5\end{array}$ & $\begin{array}{r}(410) \\
(6)\end{array}$ & $\begin{array}{r}94 \cdot 8 \\
5 \cdot 2\end{array}$ & $\begin{array}{l}(73) \\
(4)++\end{array}$ \\
\hline $\begin{array}{l}\text { Ever in drug treatment: } \\
\text { No } \\
\text { Yes }\end{array}$ & $\begin{array}{r}98 \cdot 0 \\
2 \cdot 0\end{array}$ & $\begin{array}{r}(731) \\
(15)\end{array}$ & $\begin{array}{r}96 \cdot 4 \\
3 \cdot 6\end{array}$ & $\begin{array}{r}(396) \\
(15)\end{array}$ & $\begin{array}{r}92 \cdot 1 \\
7 \cdot 9\end{array}$ & $\begin{array}{l}(70) \\
(6)+\dagger\end{array}$ \\
\hline Total & 100.0 & (775) & $100 \cdot 0$ & $(416)$ & $100 \cdot 0$ & (79) \\
\hline
\end{tabular}

IVDU $=$ intravenous drug user.
* Some variables may not sum up to $100 \%$ because of missing data.

† One sided $\chi^{2}$ test of homogeneity between telephone and face to face samples.

$\ddagger$ One sided $\chi^{2}$ test of homogeneity between telephone owners and not owners among face to face respondents.

$\S$ Question present only in a random sample of telephone questionnaires $(n=257)$.

${ }_{* *} \mathrm{p}<0.001 ; \dagger+\mathrm{p}<0.01 ; \ddagger \neq \mathrm{p}<0.05$. women in the face to face survey, in which the response rate was $77 \%$.

With regard to the demographic characteristics of women respondents (table 1), there were some significant differences between the two samples. Compared with the telephone survey respondents, the street based (face to face) sample yielded a higher proportion of women younger than 25 years $(58 \cdot 1 \% v 36 \cdot 8 \%)$, a higher proportion of unemployed women $(54.1 \% v 35 \cdot 2 \%)$, and more who lived in public housing $(56.8 \% v 37.7 \%)$. In addition, the face to face sample had a lower overall educational attainment $(62.9 \% v 79.8 \%$, respectively, reporting high school or higher education), and fewer were married $(9.2 \% v$ $21.2 \%$ ), while slightly more reported never having had sex $(8.3 \% v 4.7 \%)$. There were differences within the face to face subgroup according to telephone ownership status. Besides age and educational differences, those respondents in the face to face survey who lived in households without a telephone included more women who were not working outside home $(74.0 \%$ v $49.5 \%)$, were living in public or subsidised housing $(76.9 \% v 51.8 \%)$, had injected drugs during the last month $(5.2 \% v 0.6 \%)$, and had been in a drug treatment programme at some time $(7 \cdot 7 \% v 2 \cdot 1 \%)$.

When the telephone and the face to face samples are compared in terms of pregnancy and contraception characteristics (table 2), telephone respondents were somewhat more likely to report having had an abortion or surgical sterilisation. When stratified by age, however, statistically significant differences remained only in women aged $30-35$ years. No statistically significant differences were found by interview method in either condom use or the use of a diaphragm for contraception during the prior year.

Table 2 Pregnancy and contraception characteristics in relation to age group and questionnaire type among sexually active female respondents

\begin{tabular}{|c|c|c|c|c|c|c|c|c|c|c|}
\hline \multirow[b]{2}{*}{$\begin{array}{l}\text { Characteristic: \% } \\
\text { interview group }\end{array}$} & \multicolumn{10}{|c|}{ Age group (y) } \\
\hline & $\begin{array}{l}17-19 \\
\%\end{array}$ & (No) & $\begin{array}{l}20-24 \\
\%\end{array}$ & (No) & $\begin{array}{l}25-29 \\
\%\end{array}$ & (No) & $\begin{array}{l}30-35 \\
\%\end{array}$ & (No) & $\begin{array}{l}\text { Total } \\
\%\end{array}$ & (No) \\
\hline $\begin{array}{l}\text { Ever been pregnant: } \\
\text { Telephone } \\
\text { Face to face }\end{array}$ & $\begin{array}{l}37 \cdot 1 \\
50 \cdot 0\end{array}$ & $\begin{array}{l}(33) \\
(28)\end{array}$ & $\begin{array}{l}73 \cdot 5 \\
78.5\end{array}$ & $\begin{array}{l}(114) \\
(113)\end{array}$ & $\begin{array}{l}86 \cdot 8 \\
90 \cdot 9\end{array}$ & $\begin{array}{r}(165) \\
(90)\end{array}$ & $\begin{array}{l}87 \cdot 8 \\
90 \cdot 1\end{array}$ & $\begin{array}{r}(245) \\
(64)\end{array}$ & $\begin{array}{l}78 \cdot 2 \\
79 \cdot 7\end{array}$ & $\begin{array}{l}(557) \\
(295)\end{array}$ \\
\hline $\begin{array}{l}\text { Ever had a baby: } \\
\text { Telephone } \\
\text { Face to face }\end{array}$ & $\begin{array}{l}29 \cdot 1 \\
33 \cdot 3\end{array}$ & $\begin{array}{l}(25) \\
(17)\end{array}$ & $\begin{array}{l}63 \cdot 1 \\
71 \cdot 7\end{array}$ & $\begin{array}{l}(94) \\
(99)\end{array}$ & $\begin{array}{l}79 \cdot 8 \\
81.5\end{array}$ & $\begin{aligned}(138) \\
(66)\end{aligned}$ & $\begin{array}{l}82 \cdot 8 \\
84 \cdot 6\end{array}$ & $\begin{array}{r}(212) \\
(44)\end{array}$ & $\begin{array}{l}70 \cdot 4 \\
70 \cdot 2\end{array}$ & $\begin{array}{l}(469) \\
(226)\end{array}$ \\
\hline $\begin{array}{l}\text { Ever had an abortion* } \\
\text { Telephone } \\
\text { Face to face }\end{array}$ & $\begin{array}{r}7 \cdot 6 \\
19 \cdot 2\end{array}$ & $\begin{array}{l}(5) \\
(10)\end{array}$ & $\begin{array}{l}32 \cdot 7 \\
24 \cdot 6\end{array}$ & $\begin{array}{l}(37) \\
(32)\end{array}$ & $\begin{array}{l}37.9 \\
29 \cdot 6\end{array}$ & $\begin{array}{l}(47) \\
(24)\end{array}$ & $\begin{array}{l}43 \cdot 0 \\
23 \cdot 5\end{array}$ & $\begin{array}{l}(83) \\
(12) \S\end{array}$ & $\begin{array}{l}34 \cdot 7 \\
24 \cdot 1\end{array}$ & $\stackrel{(172)}{(78)+\dagger}$ \\
\hline $\begin{array}{l}\text { Surgical sterilisation: } \\
\text { Telephone } \\
\text { Face to face }\end{array}$ & $\begin{array}{l}0.0 \\
1.9\end{array}$ & $\begin{array}{l}(0) \\
(1)\end{array}$ & $\begin{array}{l}5 \cdot 7 \\
5 \cdot 1\end{array}$ & $\begin{array}{l}(9) \\
(7)\end{array}$ & $\begin{array}{l}27.5 \\
22.8\end{array}$ & $\begin{array}{l}(52) \\
(21)\end{array}$ & $\begin{array}{l}45 \cdot 9 \\
63 \cdot 2\end{array}$ & $\stackrel{(130)}{(43)+\dagger}$ & $\begin{array}{l}26 \cdot 4 \\
20 \cdot 6\end{array}$ & $\stackrel{(191)}{(72) \S}$ \\
\hline $\begin{array}{l}\text { Last year use of diaphragm } \dagger \\
\text { Face to face }\end{array}$ & $\begin{array}{l}1 \cdot 1 \\
0 \cdot 0\end{array}$ & $\begin{array}{l}(1) \\
(0)\end{array}$ & $\begin{array}{l}0.7 \\
2 \cdot 2\end{array}$ & $\begin{array}{l}(1) \\
(3)\end{array}$ & $\begin{array}{l}2 \cdot 7 \\
4 \cdot 1\end{array}$ & $\begin{array}{l}(5) \\
(4)\end{array}$ & $\begin{array}{l}4 \cdot 8 \\
3 \cdot 4\end{array}$ & $\begin{array}{c}(13) \\
(2)\end{array}$ & $\begin{array}{l}2 \cdot 9 \\
2 \cdot 6\end{array}$ & $\begin{array}{l}(20) \\
(9)\end{array}$ \\
\hline $\begin{array}{l}\text { Last year use of condom } \ddagger \\
\text { Telephone } \\
\text { Face to face }\end{array}$ & $\begin{array}{l}41 \cdot 6 \\
26.4\end{array}$ & $\begin{array}{l}(32) \\
(14)\end{array}$ & $\begin{array}{l}24 \cdot 1 \\
28 \cdot 7\end{array}$ & $\begin{array}{l}(33) \\
(39)\end{array}$ & $\begin{array}{l}18.9 \\
15 \cdot 3\end{array}$ & $\begin{array}{l}(30) \\
(15)\end{array}$ & $\begin{array}{r}9 \cdot 3 \\
11 \cdot 7\end{array}$ & $\begin{array}{r}(21) \\
(7)\end{array}$ & $\begin{array}{l}19.3 \\
21.6\end{array}$ & $\begin{array}{r}(116) \\
(75)\end{array}$ \\
\hline
\end{tabular}

* Totals may sum up less than those of Table 1 because of the different number of missing values. In addition,

† Question of abortion present in two thirds $(n=496)$ of the telephone questionnaires (see text).

$\ddagger$ Usually or always.

$\S \mathrm{p}<0.05 ; \dagger+\mathrm{p}<0.01$ 
Table 3 Patterns of sexual behaviour and sexual risk profile in relation to age and interview mode among sexually active female respondents

\begin{tabular}{|c|c|c|c|c|c|c|c|c|c|c|}
\hline \multirow[b]{2}{*}{$\begin{array}{l}\text { Characteristic: } \\
\text { interview group }\end{array}$} & \multicolumn{10}{|c|}{ Age group (y) } \\
\hline & $\begin{array}{l}17-19 \\
\%\end{array}$ & $(\mathrm{No})$ & $\begin{array}{l}20-24 \\
\%\end{array}$ & $(\mathrm{No})$ & $\begin{array}{l}25-29 \\
\%\end{array}$ & $(\mathrm{No})$ & $\begin{array}{l}30-35 \\
\%\end{array}$ & $(\mathrm{No})$ & $\underset{\%}{\text { Total }}$ & $(\mathrm{No})$ \\
\hline $\begin{array}{l}\text { Last sex use of } \\
\text { Telephone } \\
\text { Face to face }\end{array}$ & $\begin{array}{l}51 \cdot 2 \\
28 \cdot 6\end{array}$ & $\begin{array}{l}(44) \\
(16) \dagger\end{array}$ & $\begin{array}{l}38 \cdot 8 \\
30 \cdot 9\end{array}$ & $\begin{array}{l}(57) \\
(42)\end{array}$ & $\begin{array}{l}21 \cdot 9 \\
20 \cdot 0\end{array}$ & $\begin{array}{l}(32) \\
(16)\end{array}$ & $\begin{array}{l}21 \cdot 1 \\
33 \cdot 3\end{array}$ & $\begin{array}{r}(35) \\
(8)\end{array}$ & $\begin{array}{l}30 \cdot 8 \\
27 \cdot 7\end{array}$ & $\begin{array}{r}(168) \\
(82)\end{array}$ \\
\hline $\begin{array}{l}\text { Last sex drinkin } \\
\text { Telephone } \\
\text { Face to face }\end{array}$ & $\begin{array}{r}3 \cdot 8 \\
26 \cdot 3\end{array}$ & $\begin{array}{l}(2) \\
(15)_{+}^{+}\end{array}$ & $\begin{array}{l}16 \cdot 8 \\
25 \cdot 2\end{array}$ & $\begin{array}{l}(17) \\
(36)\end{array}$ & $\begin{array}{l}19 \cdot 5 \\
37 \cdot 0\end{array}$ & $\begin{array}{l}(24) \\
(37)_{+}^{+}\end{array}$ & $\begin{array}{l}17 \cdot 5 \\
35 \cdot 2\end{array}$ & $\begin{array}{l}(33) \\
(25)_{\ddagger}^{\ddagger}\end{array}$ & $\begin{array}{l}16 \cdot 3 \\
30 \cdot 5\end{array}$ & $\begin{array}{l}(76) \\
(113)_{\ddagger}^{\ddagger}\end{array}$ \\
\hline $\begin{array}{l}\text { Last sex using } \\
\text { Telephone } \\
\text { Face to face }\end{array}$ & $\begin{array}{l}0 \cdot 0 \\
8 \cdot 8\end{array}$ & $\begin{array}{l}(0) \\
(5) \S\end{array}$ & $\begin{array}{l}3.9 \\
9.9\end{array}$ & $\begin{array}{r}(4) \\
(14)\end{array}$ & $\begin{array}{r}3 \cdot 3 \\
11 \cdot 1\end{array}$ & $\begin{array}{l}(4) \\
(11)+\end{array}$ & $\begin{array}{r}1 \cdot 6 \\
12 \cdot 9\end{array}$ & $\begin{array}{l}(3) \\
(9) \ddagger\end{array}$ & $\begin{array}{r}2 \cdot 4 \\
10 \cdot 6\end{array}$ & $\begin{array}{l}(11) \\
(39)_{\ddagger}^{\ddagger}\end{array}$ \\
\hline $\begin{array}{l}\text { Last year two or } \\
\text { partners: } \\
\text { Telephone } \\
\text { Face to face }\end{array}$ & $\begin{array}{l}32 \cdot 2 \\
15 \cdot 8\end{array}$ & $\begin{array}{l}(28) \\
(9) \S\end{array}$ & $\begin{array}{l}28 \cdot 6 \\
24 \cdot 5\end{array}$ & $\begin{array}{l}(44) \\
(34)\end{array}$ & $\begin{array}{l}19 \cdot 1 \\
16 \cdot 7\end{array}$ & $\begin{array}{l}(36) \\
(16)\end{array}$ & $\begin{array}{l}14 \cdot 6 \\
16 \cdot 4\end{array}$ & $\begin{array}{l}(40) \\
(11)\end{array}$ & $\begin{array}{l}21 \cdot 0 \\
19 \cdot 4\end{array}$ & $\begin{array}{r}(148) \\
(70)\end{array}$ \\
\hline $\begin{array}{l}\text { Last year asked } \\
\text { STD: } \\
\text { Telephone } \\
\text { Face to face }\end{array}$ & $\begin{array}{l}56 \cdot 2 \\
25 \cdot 5\end{array}$ & $\begin{array}{l}(50) \\
(14) \dagger\end{array}$ & $\begin{array}{l}40 \cdot 6 \\
37 \cdot 8\end{array}$ & $\begin{array}{l}(63) \\
(54)\end{array}$ & $\begin{array}{l}34 \cdot 2 \\
34 \cdot 3\end{array}$ & $\begin{array}{l}(65) \\
(34)\end{array}$ & $\begin{array}{l}27 \cdot 2 \\
25 \cdot 7\end{array}$ & $\begin{array}{l}(76) \\
(18)\end{array}$ & $\begin{array}{l}35 \cdot 6 \\
32 \cdot 2\end{array}$ & $\begin{array}{l}(254) \\
(120)\end{array}$ \\
\hline $\begin{array}{l}\text { Past } 5 \text { years par } \\
\text { Telephone } \\
\text { Face to face }\end{array}$ & $\begin{array}{l}9 \cdot 1 \\
1 \cdot 8\end{array}$ & $\begin{array}{l}(8) \\
(1)\end{array}$ & $\begin{array}{l}9 \cdot 8 \\
7 \cdot 6\end{array}$ & $\begin{array}{l}(15) \\
(11)\end{array}$ & $\begin{array}{l}4 \cdot 7 \\
6 \cdot 1\end{array}$ & $\begin{array}{l}(9) \\
(6)\end{array}$ & $\begin{array}{l}2.9 \\
1 \cdot 4\end{array}$ & $\begin{array}{l}(8) \\
(1)\end{array}$ & $\begin{array}{l}5 \cdot 6 \\
5 \cdot 1\end{array}$ & $\begin{array}{l}(40) \\
(19)\end{array}$ \\
\hline
\end{tabular}

* Questions on drug and alcohol use present in two thirds ( $n=466$ for drinking; $n=458$ for tkaing drugs) of the telephone questionnaires (see text).

$\mathrm{STD}=$ sexually transmitted disease

$\dagger \mathrm{p}<0.01 ; \ddagger \mathrm{p}<0.001 ; \S \mathrm{p}<0.05$.

With regard to sexual behaviour (table 3 ) and the risk of acquiring HIV, there were only a few differences between the two samples. In respect of the use of drugs or alcohol consumption at last sexual intercourse, women were significantly more likely to respond affirmatively to these questions in the face to face survey than on the telephone. In addition, there were some consistent differences noted by interview method among women younger than 20 for most of the risk behaviours and sexual risk factors. Fewer personally interviewed respondents reported using a condom at last sexual intercourse, having two or more partners during the last year, having had a partner with a known sexually transmitted disease (STD), having asked a sexual partner about past or current STD, and having rejected sex for fear of acquiring an STD during the last year. None of these factors, however, showed any significant difference in relation to the survey mode when the samples were aggregated by age. Nor was there any statistically significant difference attributable to the data collection method for any other studied behaviour, including reported STD history in the last six months, having used illicit drugs during the last month, having ever been on a drug treatment programme, having had sexual relationship with a drug user in the past five years, or having rejected a sexual relationship because of fears of acquiring an STD during the last year.

Controlling for education, age, race, marital status, and working outside home did not change the effect of the interview mode on the reported drinking or using drugs at the last sexual encounter (table 4). However, the effects are stronger for face to face respondents who reported they did not have telephone service. These respondents had an adjusted odds ratio of $3 \cdot 7$ ( $95 \%$ confidence interval $2 \cdot 1$, 6.6) for drinking at last sexual intercourse and $14 \cdot 1(95 \%$ CI $5 \cdot 7,34.5)$ for using drugs at last sexual intercourse, compared with the telephone survey respondents. Among the subsample of face to face participants with telephone service, there remained an effect on drinking and using drugs at last sexual intercourse, at a rate approximately twice that of the telephone based sample. The differences in sterilisation (less frequent among both face to face subsamples) are not significant after adjusting for the demographic factors, while they go in the opposite direction for abortion, with an adjusted odds ratio of $0.5(95 \% \mathrm{CI} 0.4$, $0.8)$ for the telephone owner subset and 1.6 $(95 \%$ CI $0.9,2.9)$ for the non-telephone subsample.

\section{Discussion}

Our results suggest that for a inner city population of women who are mostly African American there are few differences between telephone and personal survey methods in reporting sexual behaviour. Indeed, the differences found are limited to a very specific subset of the population that can be identified for survey purposes, with telephone service at the household being particularly important. Overall, the telephone method seems particularly appropriate for less sensitive issues of relevance to HIV preventive intervention efforts or family planning programmes, such as information on pregnancy history. However, the response rate was lower among telephone respondents compared with the face to face survey. Also, we found some differences in some particularly risky behaviours, such as 
Table 4 Relationship between interview mode and telephone ownership and selected reported risk behaviour for sexually active female respondents

\begin{tabular}{|c|c|c|c|c|c|c|c|c|c|c|c|c|c|c|c|c|}
\hline \multirow[b]{2}{*}{ Interview mode } & \multicolumn{3}{|c|}{ Drinking at last sex } & \multicolumn{4}{|c|}{ Using drugs at last sex } & \multicolumn{4}{|l|}{ Sterilisation } & \multicolumn{4}{|c|}{ Ever had an abortion } & \multirow[b]{2}{*}{$\begin{array}{l}\operatorname{Adj} O R \\
(95 \% C I)\end{array}$} \\
\hline & $\%$ & $(\mathrm{No})^{*}$ & $\begin{array}{l}\text { OR } \\
(95 \% C I)\end{array}$ & $\begin{array}{l}\text { Adj OR† } \\
(95 \% C I)\end{array}$ & $\%$ & $(\mathrm{No})^{*}$ & $\begin{array}{l}\text { OR } \\
(95 \% C I)\end{array}$ & $\begin{array}{l}\text { Adj OR† } \\
(95 \% C I)\end{array}$ & $\%$ & $(\mathrm{No})^{*}$ & $\begin{array}{l}O R \\
(95 \% C I)\end{array}$ & $\begin{array}{l}\text { Adj OR† } \\
(95 \% C I)\end{array}$ & $\%$ & $(\mathrm{No})^{*}$ & $\begin{array}{l}\text { OR } \\
(95 \% C I)\end{array}$ & \\
\hline $\begin{array}{l}\text { Telephone } \\
(\mathrm{n}=716)\end{array}$ & $16 \cdot 3$ & $(76)$ & 1 & 1 & $2 \cdot 4$ & (11) & 1 & 1 & $26 \cdot 6$ & (189) & 1 & 1 & $35 \cdot 5$ & $(161)$ & 1 & 1 \\
\hline $\begin{array}{l}\text { Face to face } \\
\text { telephone owners } \\
(n=296)\end{array}$ & $27 \cdot 2$ & $(80)$ & $\begin{array}{l}1.9 \\
(1 \cdot 4-2 \cdot 8)\end{array}$ & $\begin{array}{l}1 \cdot 8 \\
(1 \cdot 2-2 \cdot 6)\end{array}$ & $6 \cdot 1$ & (18) & $\begin{array}{l}2 \cdot 7 \\
(1 \cdot 3-5 \cdot 8)\end{array}$ & $\begin{array}{l}2 \cdot 3 \\
(1 \cdot 0-5 \cdot 3)\end{array}$ & $21 \cdot 1$ & $(57)$ & $\begin{array}{l}0 \cdot 7 \\
(0 \cdot 5-1 \cdot 0)\end{array}$ & $\begin{array}{l}1 \cdot 0 \\
(0 \cdot 6-1 \cdot 5)\end{array}$ & $20 \cdot 5$ & (53) & $\begin{array}{l}0.5 \\
(0.3-0.7)\end{array}$ & $\begin{array}{l}0.5 \\
(0.4-0.8)\end{array}$ \\
\hline $\begin{array}{l}\text { Face to face } \\
\text { no telephone } \\
\text { owners }(n=72)\end{array}$ & $44 \cdot 4$ & (32) & $\begin{array}{l}4 \cdot 1 \\
(2 \cdot 4-7 \cdot 0)\end{array}$ & $\begin{array}{l}3 \cdot 7 \\
(2 \cdot 1-6 \cdot 6)\end{array}$ & $27 \cdot 8$ & $(20)$ & $\begin{array}{l}16 \cdot 0 \\
(7 \cdot 3-35 \cdot 2)\end{array}$ & $\begin{array}{l}14 \cdot 1 \\
(5 \cdot 7-34 \cdot 5)\end{array}$ & $21 \cdot 4$ & $(15)$ & $\begin{array}{l}0.8 \\
(0 \cdot 4-1 \cdot 4)\end{array}$ & $\begin{array}{l}0 \cdot 9 \\
(0 \cdot 4-1 \cdot 7)\end{array}$ & $44 \cdot 6$ & (25) & $\begin{array}{l}1.5 \\
(0.8-2.9)\end{array}$ & $\begin{array}{l}1 \cdot 6 \\
(0 \cdot 9-2 \cdot 9)\end{array}$ \\
\hline
\end{tabular}

$\mathrm{OR}=$ odds ratio; Adj $\mathrm{OR}=$ adjusted odds ratio; $95 \% \mathrm{CI}=95 \%$ confidence interval.

mat mot up to the table 2 totals because of different numbers missing for each variable.

† Odds ratio adjusted by age, education, employment outside home and marital status.

consumption of alcohol and drugs at last sexual intercourse. These may be considered proxy measures of other risk behaviours for which we did not find any difference, thus raising concerns on the direct comparability of these two survey methods.

Methodological issues stemming from the comparison of telephone and face to face surveys are numerous and complex. As Groves ${ }^{3}$ points out, the ideal "experimental" comparison of telephone and face to face methods would include identical questions, measures balanced in their appropriateness to both method, identical interviewers with equivalent experience, identical population samples, respondents unaffected by previous exposure to one or the other mode, to name but a few of the theoretical criteria of comparability. However, empirical evidence of differences between these methods comes from studies in which the element of comparison is not only the method but a constellation of all other elements, including the wording, length of survey, and skills of interviewer. Some of these effects were partially overcome in this study since the same interviewers carried out both surveys. However, the possibility of both a selection and a method effect remain, and to some extent our data suggest that both account for the observed differences.

With regard to the demographic composition of samples obtained in the telephone and personal interviews, differences between the two are striking, although similar to findings in previous studies. ${ }^{35}$ Refusal and recruitment methods might have added to the selection bias in the street sample, since obviously threatening or uncooperative respondents were not approached or failed to conduct the interview. While the non-response rate for the telephone survey $(33.6 \%)$ was not higher than expected in telephone surveys, ${ }^{12}$ the response bias may well account for some of the observed differences. Groves, ${ }^{18}$ in a follow up of initial non-respondents to a telephone survey, found that these people tended to be younger and were less often married. We have observed the same trend, since the proportion of respondents younger than 25 was lower in the telephone $(36.8 \%)$ than in the face to face survey
$(58 \cdot 1 \%)$. While this bias hampers direct comparison of the samples, it is partly offset when comparisons of the responses are made within five years intervals. Although the response rates related to each method are not directly comparable, $23 \%$ of the potential resondents approached on the street refused. The response bias associated with the face to face, street intercept sampling, has not been extensively studied. Since the characteristics of nonrespondents were not ascertained, the direction of bias is not know, although its impact is presumably smaller since the proportion of non-respondents was much lower than in the telephone survey. However, it is important to consider that since there was no sampling frame for the face to face survey, the refusal rate is only a tentative estimate, and can not be directly compared with the telephone survey response.

The survey method effect can be explained in terms of under-reporting of sensitive issues in a telephone interview. In this regard, our results are consistent with previous studies that have found only small differences between the two methods in assessing sensitive topics. ${ }^{23719-22}$ Nevertheless, it has been reported that less wealthy respondents are more prone to the "social desirability effect" in telephone surveys. ${ }^{3}$ Aquilino ${ }^{23}$ reported a lower reporting of alcohol and marijuana use among African-Americans interviewed by telephone than in those interviewed face to face, differences that did not hold for white respondents. To explain these differences, Cain ${ }^{5}$ claims that the interviewer is unlikely to develop sufficient rapport over the telephone to gather high quality data on sensitive topics. Catania $^{6}$ has suggested that there are several reasons for suspecting inconsistencies between people's actual sexual behaviour and their self reports in an interview-privacy, embarrassment, and fear of reprisals may lead some to mask the facts, while others may, on the contrary, magnify their actual sexual performance or experience. Of control importance to surveys assessing sexual practices are differences in the degree of privacy extended to respondents as they answer questions about sexual behaviour, and the degree of credibility the 
study's representatives achieve with regard to respondents' trust in the legitimate purpose of the questions concerning sexual behaviour. Previous findings of differences associated with the method can be explained by these factors. Bradburn ${ }^{24}$ describes a higher proportion of respondents affirming that they had been driving after drinking alcohol, in telephone than in fact to face interviews. McQueen ${ }^{9}$ found a higher proportion who declared risk behaviour, such as a greater number of partners or homosexual relationships, among telephone than face to face respondents. Hochstim ${ }^{19}$ reported that $10 \%$ fewer respondents reported any alcohol consumption during telephone interview compared with face to face interview, when white, middle class, middle aged women were approached. Schober ${ }^{25}$ who reinterviewed 10307 respondents to the 1988 National Longitudinal Survey of Labor Market Experience reported that for white and hispanic populations, self administered questionnaires gave higher reports of drug use, which was not true for African-American respondents. In our study, the lack of privacy related to the fact that interviewing took place in the street does not seem to have played a significant role, since none of the behaviours that can be socially considered as "risky" (for example, a higher number of partners) was reported less often by the face to face respondents. This may be partly explained by the high prevalence of these behaviours in these two communities.

The observed differences in the proportion, who declared they had had an abortion or had been sterilised are partly related to the demographic differences in the samples. Thus, the differences in sterilisation dissappear after adjusting for sociodemographic factors, while a small effect remains regarding abortions, which are less likely to be reported in the face to face sample. While these behaviours are not directly related to the risk of acquiring HIV infection, they can influence the occurrence of other risk factors: sterilised women in our study were to be less likely to use condoms, ${ }^{26}$ which is obviously related to the lack of contraceptive motivation. On the other hand, it can be argued that the past experience of abortions can influence the probability of terminating a pregnancy in the presence of HIV infection.

Most risk behaviours are more frequently reported by adolescent telephone respondents than by their face to face counterparts. The finding is consistent with Turner's ${ }^{27}$ report from the 1990 National Household Survey on Drug Abuse that adolescents were more sensitive than older respondents to the lack of intimacy when answering questions about use of alcohol and drugs in a personal interview. It is likely that for the youngest respondents, embarrassment and lack of privacy can lead to under-reports of undesirable behaviour in the personal interview, a form of the cultural self presentation bias, described by Catania. ${ }^{6}$ Nevertheless, this difference raises the concern that for this particular age group, the standard survey methods, and particuarly telephone surveys, are not appropriate when exploring issues that may be particularly sensitive.

Overall, the evidence supports the conclusion that we can use, within certain limits, telephone surveys as a cost effective way of estimating at the community level certain behaviours, including contraception and sexual behavioural. This may be particularly relevant to the need to monitor risk factors related to HIV infection. In addition, telephone interviews may be more accurate for adolescents, since they seem less likely to report some sexual behaviours in the face to face interview. However, telephone surveys may not be appropriate for determining HIV risk factors, specially sexual behaviour, in some cultural contexts in which these topics are considered quite sensitive. Also, it becomes apparent that when approaching socially and economically deprived communities, we have to be aware of the selection bias of a telephone survey, that will presumably miss the highest risk respondents. Nevertheless, the bias can be partly accounted for by proxy indicators of socioeconomic status. While it seems plausible that telephone respondents under-report some sensitive issues, the question of the magnitude of bias in a particular population must be addressed before making inferences from a survey, disregarding the method. In some cases, qualitative research can be necessary to assess the cultural barriers to self report, providing additional understanding to quantitative data obtained through survey methods.

1 Lavrakas PJ. Telephone survey methods. Sage: New York, 1987.

2 Rogers TF. Interviews by telephone and in person: quality of responses and field performance. Public Opinion Quarterly 1976; 40: 51-65.

3 Groves RM, Kahn RL. Surveys by telephone. A national comparison with personal interviews. New York: Academic comparison with person.

4 Schuman H, Presser S. Questions and answers in attitude surveys. Experiments on question form, wording and context. New York: Academic Press, 1981 .

5 Cain VS. Methodological experiments in the National Survey of Health and Sexual Behavior. In: Fowler FJ. Health survey research methods. Conference Proceedings. Rockville: National Center for Health Services Research and Health Care Technology Assessment. Department of Health and Human Services no (PHS) 893447, 1989: 2415.

6 Catania JA, Coates TJ, Stall R, et al. Prevalence of AIDSrelated risk factors and condom use in the United States. Science 1992; 258: 1101-6.

7 Coombs L, Freedman R. Use of telephone interviews in a longitudinal fertility study. Public Opinion Quarterly 1964; 28: 112-7.

8 Czaja R. Asking sensitive behavioral questions in telephone interviews. International Quarterly of Community Health Education 1987; 8: 233-2.

9 McQueen DV. Comparison of results of personal interview and telephone surveys of behavior related to risk of AIDS: and telephone surveys of behavior related to risk of AIDS: advantages of telephone techniques. In: Fowler FJ. Health Survey Research Methods. Conference Proceedings. Rock-
ville: National Center for Health Services Research and ville: National Center for Health Services Research and
Health Care Technology Assessment. Department of Health and Human Services no. (PHS) 893447, 1989: 247252 .

10 Cinamon ML, Kronenfeld JJ, Laumann EO. Measuring behavior related to risk of AIDS. Session summary. In: Fowler FJ. Health Survey Research Methods. Conference Proceedings, Rockville: National Centre for Health Services Research and Health Care Technology Assessment. Department of Health and Human Services no. (PHS) 893447: 263-264.

11 National Center for Health Statistics. Report of final natality statistics, 1987. Monthly Vital Statistics Report. 1988 (suppl 3): 38

12 Augustyn $M$. Condom use in an urban population: an application of the theory of Planned Behavior. Baltimore: John

13 Nunally JC. Psychometric theory. New York: McGraw Hill, 1978: 35-85.

14 Waksberg J. Sampling methods for random digit dialing fournal of the American Statistical Association 1978; 78 $(361): 40-6$ 
15 Watters JK, Biernacki P. Targeted sampling: options for the study of hidden populations. Social Problems $1989 ; 36$ 416-30.

16 Fleiss JL. Statistical methods for rates and proportions. New York: Wiley, 1981.

17 Hosmer DW, Lemeshow S, Applied logistic regression. New York: John Wiley \& Sons, 1989: 58-63.

18 Groves RM. An empirical comparison of two telephone sample designs. Fournal of Marketing Research 1978; 25 622-31.

19 Hochstim J. A critical comparison of three strategies of collecting data from households. Fournal of the American Statistical Association 1967; 62: 976-89.

20 Kegeles S, Fink C, Kirscht J. Interviewing a national sample by long distance telephone. Public Opinion Quarterly 1969; 33: 412-19.

21 San Francisco AIDS Foundation. Designing an effective AIDS risk reduction program for San Francisco: results AIDS risk reduction program for San Francisco: results
from the first probability sample of multiple/high risk partner heterosexual adults. San Francisco: Communication Techneterosexual adults. San Francisco: Communication

22 Levy J, Albrecht G. Methodological considerations in research on sexual behavior and AIDS among older people.
In: Riley $\mathrm{M}$, Ory $\mathrm{M}$, Zablotsky $\mathrm{D}$ (ed). AIDS in an aging society: what we need to know. New York: Springer, 1989. 23 Aquilino WS, LoSciuto LA. Effects of mode of data collection on the validity of reported drug use. In: Fowler FJ. Health survey research methods. Conference Proceedings. Rockville: National Center for Health Services Research and Health Care Technology Assessment. Department of Health and Human Services publication no. (PHS) 893447, 1989: 253-8.

24 Bradburn N, Sudman S. Improving interview method and questionnaire design. San Francisco: Jossey Bass, 1979.

25 Schober S, Caces MF, Pergamit M, Branden L. Effect of mode of administration on reporting of drug use in the National Longitudinal Survey. In: Turner CF, Lessler JT Gfroerer JC eds. Survey measurement of Lessler JT, Groerer JC eds. Survey measurement of drug use. tute on Drug Abuse, 1992.

26 Santelli JS, Burwell LG, Rozsenich C, Augustyn M, Rolf JE, Wallach R, Beverly. $M M W R$ 1992; 41: 573-5.

27 Turner CF, Lessler JT, Gfroerer JC eds. Survey measurement of drug use. Methodological studies. Rockville (MD): National Institute on Drug Abuse. DHSS publication no (adm) 921929, 1992. 